\title{
The Mid-IR Extinction Law in the LMC
}

\author{
Jian Gao, Mengyao Xue, and B. W. Jiang \\ Department of Astronomy, Beijing Normal University, \\ Beijing 100875, China \\ email: jiangao@bnu.edu.cn
}

\begin{abstract}
The mid-infrared (MIR) extinction law in the Large Magellanic Cloud (LMC) at four IRAC bands is derived using the data of the Spitzer/SAGE Program. The derived mean extinctions are $A_{[3.6]} / A_{\mathrm{K}_{\mathrm{s}}}=0.68 \pm 0.03, A_{[4.5]} / A_{\mathrm{K}_{\mathrm{s}}}=0.97 \pm 0.03, A_{[5.8]} / A_{\mathrm{K}_{\mathrm{s}}}=0.54 \pm 0.04$, and $A_{[8.0]} / A_{\mathrm{K}_{\mathrm{s}}}=0.58 \pm 0.07$. The results show that: (1) The extinctions at [3.6], [5.8] and [8.0] of the LMC consist a flat curve, similar to that of the Milky Way (MW) predicted by the interstellar grain model at $R_{\mathrm{V}}=5.5 ;$; 2 ) The extinction at [4.5] is clearly higher than the other three bands, which may be caused by the additional absorption of the $4.27 \mu \mathrm{m} \mathrm{CO} 2$ ice and/or the $4.67 \mu \mathrm{m}$ $\mathrm{CO}$ ice in the LMC molecular clouds; (3) As far as individual sightlines are concerned, the MIR interstellar extinction law $A_{\lambda} / A_{\mathrm{K}_{\mathrm{s}}}$ in the LMC varies with sightlines as the $\mathrm{MW}$ does.
\end{abstract}

Keywords. dust, extinction — infrared: ISM — Magellanic Clouds

In the UV and V bands, the LMC has an average extinction curve characterized by a weaker $2175 \AA$ bump and a stronger far-UV rise than the MW. Many studies have shown that regional variations also exist in the LMC extinction law. In the near-infrared (NIR), Koornneef (1982) derived the extinction law in the LMC and argued that it is very similar to that of the MW. Imara \& Blitz (2007) obtained the NIR extinction coefficients $\left[E(\mathrm{~J}-\mathrm{H}) / E\left(\mathrm{H}-\mathrm{K}_{\mathrm{s}}\right)=1.20 \pm 0.04\right]$ of the LMC, consistent with that of Koornneef (1982). On the other hand, the information of the MIR extinction is absent for the LMC. In this work, we probed the MIR extinction law of the LMC based on the Spitzer/SAGE's data.

The mean $A_{\mathrm{V}}$ of the LMC is about $0.38 \mathrm{mag}$ (Imara \& Blitz 2007), which means the MIR extinction would be very small $\left(A_{\mathrm{K}_{\mathrm{s}}} \sim 0.1 A_{\mathrm{V}}\right)$. However, the maximum $A_{\mathrm{V}}$ of the LMC is about $5.0 \mathrm{mag}$, implying that the MIR extinction is significant and necessary to correct for. Therefore, we chose five fields with large $A_{\mathrm{V}}(>2 \mathrm{mag}$ ) according to the extinction map of Dobashi et al. (2008). The tracers for extinction are red giants (RGBs) (Gao et al. 2009). They ( 8,127 sources) are selected from the Nikolaev \& Weinberg (2000)'s Region $E$ in the 2MASS Color-Magnitude Diagram, i.e. $12<\mathrm{K}_{\mathrm{s}}<13.5$ and $0.9<\mathrm{J}-\mathrm{K}_{\mathrm{s}}<1.2$, covering the upper RGBs and the tip of the RGBs. With $R_{\mathrm{V}}=3.01$, $A_{\mathrm{J}} / A_{\mathrm{K}_{\mathrm{s}}}=3.28$ is derived from the NIR extinction law by Koornneef (1982). The MIR extinction law is calculated by using the color-excess method. In addition, the diagram of $\mathrm{J}-\mathrm{H}$ v.s. $\mathrm{H}-\mathrm{K}_{\mathrm{s}}$ is fitted to derive the NIR extinction law: $E(\mathrm{~J}-\mathrm{H}) / E\left(\mathrm{H}-\mathrm{K}_{\mathrm{s}}\right)=$ $1.27 \pm 0.04$, which is consistent with that of Imara \& Blitz (2007) and Koornneef (1982).

Our main results are summarized in the abstract above.

\section{References}

Dobashi, K., Bernard, J.-P., Hughes, A., et al. 2008, A\&SA 484, 205

Gao, J., Jiang, B. W., \& Li, A. 2009, ApJ 707, 89

Imara, N. \& Blitz, L. 2007, ApJ 662, 969

Koornneef, J. 1982, A\&SA 107, 247

Nikolaev, S. \& Weinberg, M. D. 2000, ApJ 542, 804 